\title{
High energy neutrinos from choked GRBs and their flavor ratio measurement by the IceCube
}

\author{
Karla Varela ${ }^{1,3}$, Sarira Sahu ${ }^{2, a}$, Andrés Felipe Osorio Oliveros ${ }^{1}$, Juan Carlos Sanabria ${ }^{1}$ \\ ${ }^{1}$ Universidad de Los Andes, Bogota, Colombia \\ 2 Instituto de Ciencias Nucleares, Universidad Nacional Autónoma de México, Circuito Exterior, C.U., \\ A. Postal 70-543, 04510 Mexico, DF, Mexico \\ ${ }^{3}$ Max-Planck-Institut fur Extraterrestrische Physik, Giessenbachstrasse 1, 85748 Garching, Germany
}

Received: 30 November 2014 / Accepted: 16 June 2015 / Published online: 26 June 2015

(C) The Author(s) 2015. This article is published with open access at Springerlink.com

\begin{abstract}
The high energy neutrinos produced in a choked gamma-ray burst can undergo matter oscillation before emerging out of the stellar envelope. Before reaching the detector on Earth, these neutrinos can undergo further vacuum oscillation and then Earth matter oscillation when crossing the diameter of the Earth. In the context of IceCube we study the Earth matter effect on neutrino flux in the detector. For the calculation of the track-to-shower ratio $R$ in the IceCube, we have included the shadowing effect and the additional contribution from the muon track produced by the high energy tau lepton decay in the vicinity of the detector. We observed that $R$ is different for different CP phases in vacuum but the matter effect suppresses these differences. We have also studied the behavior of $R$ when the spectral index $\alpha$ varies.
\end{abstract}

\section{Introduction}

Gamma-ray bursts (GRBs) are cosmological events with the emission of very intense electromagnetic radiation in the energy range $\sim 100 \mathrm{keV}-1 \mathrm{MeV}$. Phenomenologically GRBs come in two variants: the short-hard bursts and long-soft bursts. The long gamma-ray bursts (LGRBs, typically with duration longer than $2 \mathrm{~s}$ ), which constitute about $3 / 4$ of the total observed GRBs, are generally believed to be associated with deaths of massive stars [1,2]. In this scenario the gamma rays emitted by the collapsing star during a long GRB event should be the result of relativistic jets of radiation and matter breaking through the stellar envelope. Fermi-accelerated electrons would produce gamma rays by synchrotron and inverse Compton scattering in optically thin magnetized relativistic shocks. In this same shock protons should also be

\footnotetext{
a e-mail: sarira@nucleares.unam.mx
}

accelerated to relativistic velocities and interact with the photons producing neutrinos with an energy range from $\mathrm{MeV}-$ $\mathrm{EeV}[3,4]$. Observationally, only a small fraction $\left(\leq 10^{-3}\right)$ of core collapse $\mathrm{SNe}$ are associated with GRBs [5-7]. These correspond to the cases when the energetic jet successfully penetrates through the stellar envelope and reaches a highly relativistic speed (Lorentz factor $\Gamma \geq 100$ ). It is possible that the larger fraction of the core collapse may not be able to punch through the massive envelope to launch a successful GRB. Irrespective of its failure to emerge from the thick envelop, like the successful jet, these choked jets can also accelerate protons to very high energy and produce multi-TeV neutrinos through interaction with the keV photon background present in the jet environment [8]. The high energy neutrinos are produced from the decay of charged pions which lead to the neutrino flux ratio at the source $\Phi_{\nu_{e}}^{0}: \Phi_{v_{\mu}}^{0}: \Phi_{\nu_{\tau}}^{0}=1: 2: 0\left(\Phi_{v_{\alpha}}^{0}\right.$ corresponds to the sum of neutrino and antineutrino flux at the source). As is well known, the matter effect can substantially modify the flux ratio due to neutrino oscillation, in a presupernova star scenario; high energy neutrinos propagating through a heavy envelope can oscillate to other flavors due to matter effects, resulting in flavor ratios at the surface of the star that can be significantly different from 1:2:0. In a previous paper [9] (Paper I) we presented a detailed calculation of the effects of matter inside the presupernova star on the neutrino fluxes, using a formalism that takes into account the three neutrino flavors and different density profiles for the presupernova star. Our results show that for neutrinos with $E_{v} \leq 10 \mathrm{TeV}$ the fluxes on the surface of the star are different from the original one 1:2:0. We have also calculated the fluxes of these neutrinos on the surface of the Earth after they travel through the long baseline between the source and the Earth. We found that for neutrino energy $E_{v} \leq 10 \mathrm{TeV}$, the flux ratio is different from 1:1:1 and above this energy the ratio converges to $1: 1: 1$ implying 
that the matter effect does not play a significant role for high energy neutrinos.

The IceCube neutrino detector in South pole is fully operational since December 2010. The IceCube collaboration has reported the observation of 37 neutrino events in the energy range $30 \mathrm{TeV}-2 \mathrm{PeV}$ and the sources of these events are unknown [10-12]. These neutrino events have flavors, directions, and energies not compatible with the atmospheric neutrinos and it is believed that this is the first indication of the extraterrestrial origin of high energy neutrinos. Recently, the IceCube collaboration has presented results of 641 days data taken during 2010-2012 in the energy range $1 \mathrm{TeV}-$ $1 \mathrm{PeV}$ from the southern sky, which gives a new constraint on the diffuse astrophysical neutrino spectrum [13]. These high energy neutrino events have generated much interest and several models have been proposed as to their origin. The choked GRBs are potential candidates to produce the high energy neutrinos which can propagate hundreds of Mpc baseline to reach the Earth. So it is important to study these neutrinos and the matter effect on their propagation. The present work is an extension of Paper I. Here we take into account the matter effect of both the presupernova star medium and the Earth on the calculation of the flux ratio by a detector like IceCube, which could be relevant to get information regarding the type of progenitor responsible for the choked GRBs. Both the matter effect and the shadowing effect are important only for the upward going neutrinos. So here we take into account these effects on the neutrinos.

The organization of the papers is as follows: In Sect. 2 we discuss the neutrino propagation in the Earth by considering a realistic density profile. Here we also take into account the shadowing effect which is important for high energy neutrinos. In Sect. 3, the signature of shower and track events are discussed. The detailed calculation of track-to-shower ration is discussed in Sect. 4. Finally we present our results in Sect. 5 followed by a summary in Sect. 6 .

\section{Matter effect on neutrinos going through the Earth}

The energy spectra of the gamma rays produced by long GRBs have been measured and they follow power laws, or broken power laws [14]. In the GRB jet (both successful and choked), neutrinos are produced with varying energy depending on the distance from the central engine. The ones which are closer to the central engine are in the $\mathrm{MeV}$ range and this increases as the distance increases. This happens because the protons are Fermi accelerated within the jet and gain energy as the distance increases up to a maximum, where neutrinos of $\sim \mathrm{EeV}$ energy can be produced. In this environment the high energy $\gamma$-rays and neutrinos are produced through $p p$ and/or $p \gamma$ interaction within the jet environment and the fluxes of these $\mathrm{GeV}-\mathrm{TeV}$ neutrinos and the $\gamma$-rays are related.
Both the $\gamma$-rays and the neutrinos have a power-law spectrum. Here we assume a simple power-law spectrum for the high energy neutrinos:

$\frac{\mathrm{d} F_{\nu_{l}}}{\mathrm{~d} E_{v_{l}}}=N_{v_{l}} E_{v_{l}}{ }^{-\alpha}$,

where $\alpha \geq 2$ is the spectral index and $N_{v_{l}}$ is the normalization constant in units of $\mathrm{GeV}^{-1} \mathrm{~cm}^{-2} \mathrm{~s}^{-1}$.

High energy neutrinos reaching the detector on Earth from the opposite side can experience both oscillation absorption due to neutrino-nucleon $\mathrm{CC}$ and to $\mathrm{NC}$ interactions. The oscillation is important for the low energy neutrinos $E_{v} \leq 10$ $\mathrm{TeV}$, and the effective potential the neutrinos will experience is $V=\sqrt{2} G_{\mathrm{F}} n_{\mathrm{e}}$, where $G_{\mathrm{F}}$ is the Fermi coupling constant and $n_{\mathrm{e}}$ is the electron density in the medium. For very high energy neutrinos the interaction cross sections are large enough so that the absorption effects become very important and have to be taken into account. The shadowing factor due to this absorption is given by [15]

$P_{\text {shad }}=\exp \left(-N_{\mathrm{A}} \sigma_{T O T} X\right)$,

where $\sigma_{T O T}$ is the total neutrino-nucleon cross section, $N_{\mathrm{A}}=$ $6.0221 \times 10^{23} \mathrm{~mol}^{-1}$ is Avogadro's number, and $X$ is the column depth traveled by the neutrino inside the Earth before interaction. The column depth is the product of the distance traveled and the density of matter inside the Earth $\rho_{\mathrm{e}}$. Since the Earth's density depends on the position, $\rho_{\mathrm{e}}=\rho_{\mathrm{e}}(r)$ and $X$ is given by

$X=\int \rho_{\mathrm{e}}(r) \mathrm{d} \mathbf{r}$

where the integral is a path integral along the trajectory of the neutrino, from the entrance point to the Earth up to the detector, and can be parameterized in terms of the zenith angle $\theta$ of the neutrino track at the detector. The cross section $\sigma_{T O T}$ is a function of the neutrino energy $E_{\nu}$. Then the shadowing factor $P_{\text {shad }}$ depends on both $E_{v}$ and $\theta$ and can be expressed as $P_{\text {shad }}=P_{\text {shad }}\left(E_{\nu}, \theta\right)$. We consider the most realistic density profile of the Earth, which is given by [15]

$$
\rho_{\mathrm{e}}(r)= \begin{cases}13.0885-8.8381 \cdot x^{2} & x<0.192, \\ 12.5815-1.2638 \cdot x- & \\ 3.6426 \cdot x^{2}-5.5281 \cdot x^{3} & 0.192<x<0.546, \\ 7.9565-6.4761 \cdot x+ & \\ 5.5283 \cdot x^{2}-3.0807 \cdot x^{3} & 0.546<x<0.895, \\ 5.3197-1.4836 \cdot x & 0.895<x<0.906, \\ 11.2494-8.0298 \cdot x & 0.906<x<0.937, \\ 7.089-3.8045 \cdot x & 0.937<x<0.965, \\ 2.691+0.6924 \cdot x & 0.965<x<0.996, \\ 2.9 & 0.996<x<0.998, \\ 2.6 & 0.998<x<0.999 \\ 1.02 & 0.999<x \leq 1,\end{cases}
$$




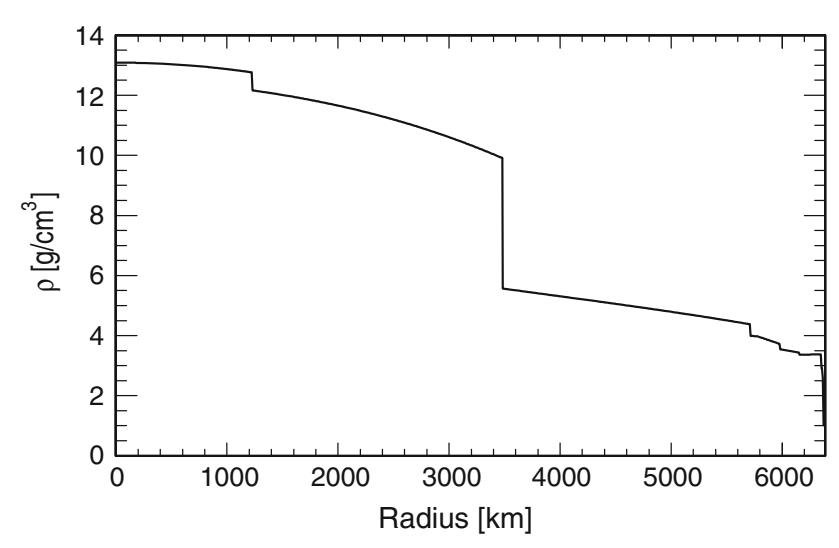

Fig. 1 Earth matter density profile $\rho_{e}\left(\mathrm{~g} / \mathrm{cm}^{3}\right)$ as a function of the radius

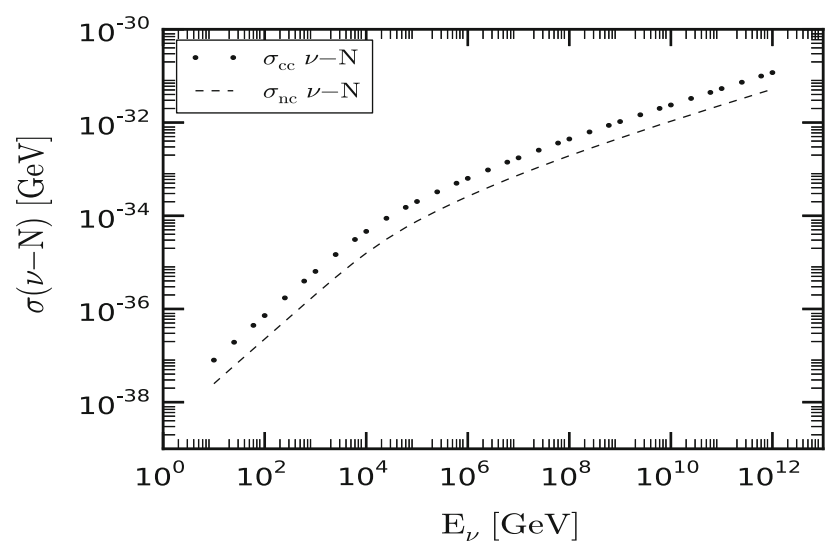

Fig. 2 Neutrino-nucleon cross sections at high energies [16]

where $x=r / R_{\text {Earth }}$ and $\rho_{\mathrm{e}}$ is given in units of $\mathrm{g} / \mathrm{cm}^{3}$. The Earth density profile is shown in Fig. 1. Using this density profile $X(\theta)$ can be calculated.

The values of the total cross sections, for neutrino and antineutrino interaction with matter (nuclei) at high energies, have to be extrapolated from low energy data, since no measurements have been performed yet. In this work we use the cross sections reported in Ref. [16] and present in Figs. 2 and 3, respectively, for $v-N$ and $\bar{v}-N$. Comparison of the total cross sections $v-N$ and $\bar{v}-N$ shows that in the low energy limit $E_{v} \leq 10 \mathrm{TeV}$ there is a very small difference between these two which can be seen in Fig. 4.

In Fig. 5, $P_{\text {shad }}$ is plotted as a function of $E_{v}$, for a zenith angle $\theta=180^{\circ}$ (neutrinos arriving to the detector from underneath). From the graph it can be noticed that the shadowing factor decreases as the neutrino energy increases beyond $\sim 1 \mathrm{TeV}$ and the Earth becomes opaque for neutrinos with energies above $\sim 1000 \mathrm{TeV}$. There is a small difference between the neutrino and antineutrino shadowing factor above $1 \mathrm{TeV}$. Since we are interested in $\mathrm{TeV}$ neutrinos, the shadowing effect has to be taken into account properly in the calculation of the neutrino fluxes arriving

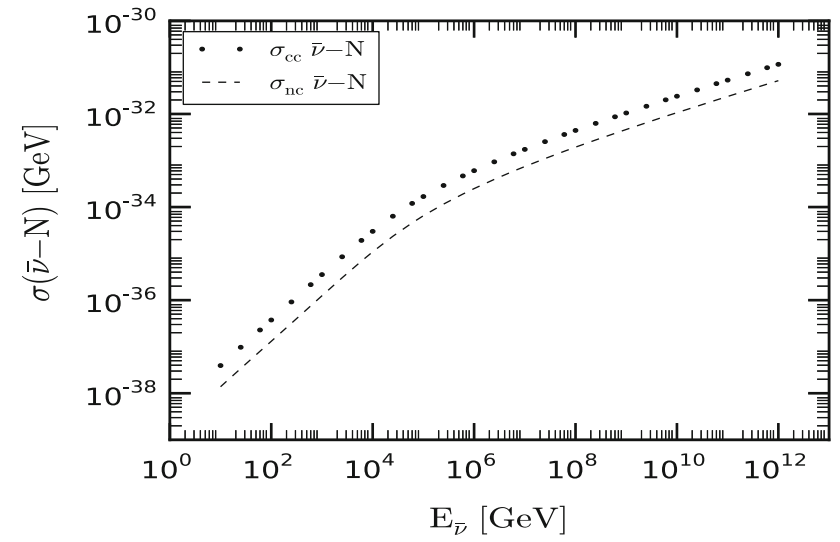

Fig. 3 Antineutrino-nucleon cross sections ant high energies [16]

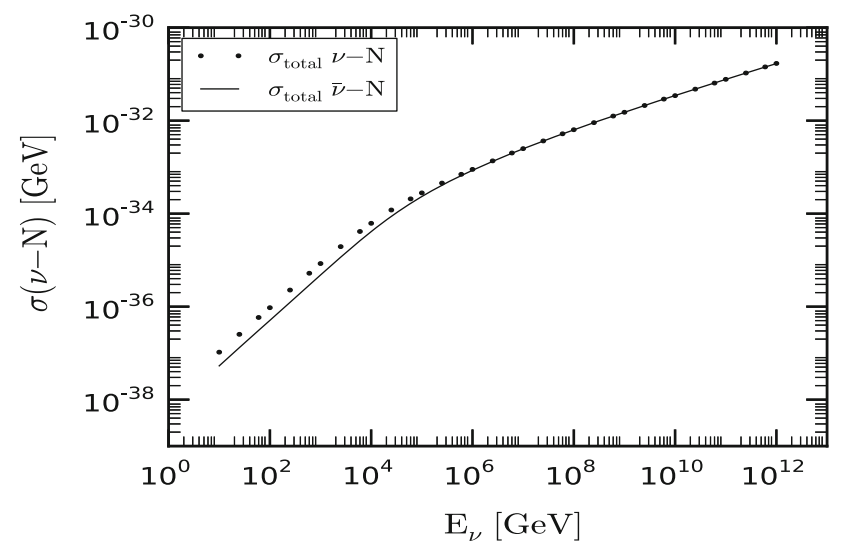

Fig. 4 Comparison of both neutrino-nucleon and antineutrinonucleon cross sections

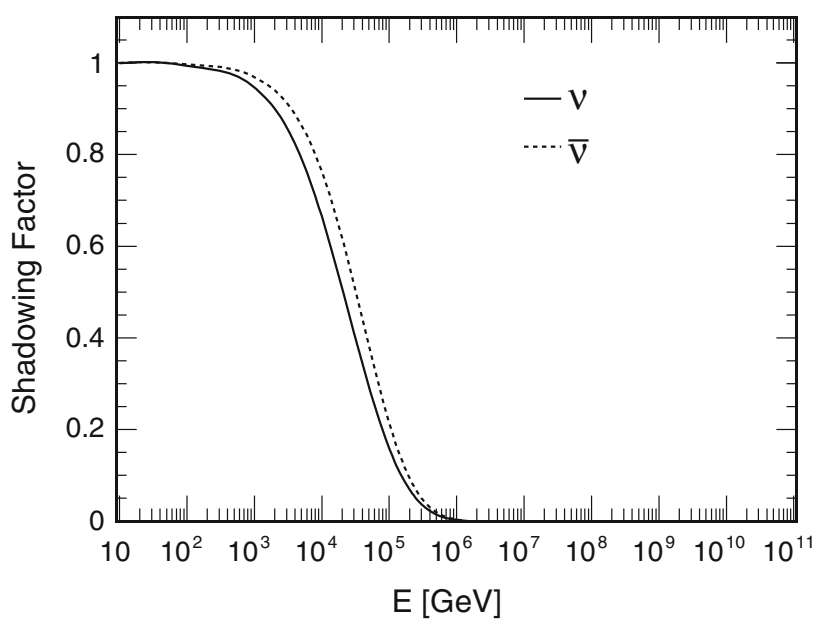

Fig. 5 The shadowing factor $P_{\text {shad }}$ as a function of the neutrino energy for a zenith angle $\theta=180^{\circ}$

at the detector. Depending on the energy of the neutrinos, the interaction of the neutrinos with the medium inside the Earth will also result in flavor oscillations. Since in this work we will account for those neutrinos that go through the Earth 


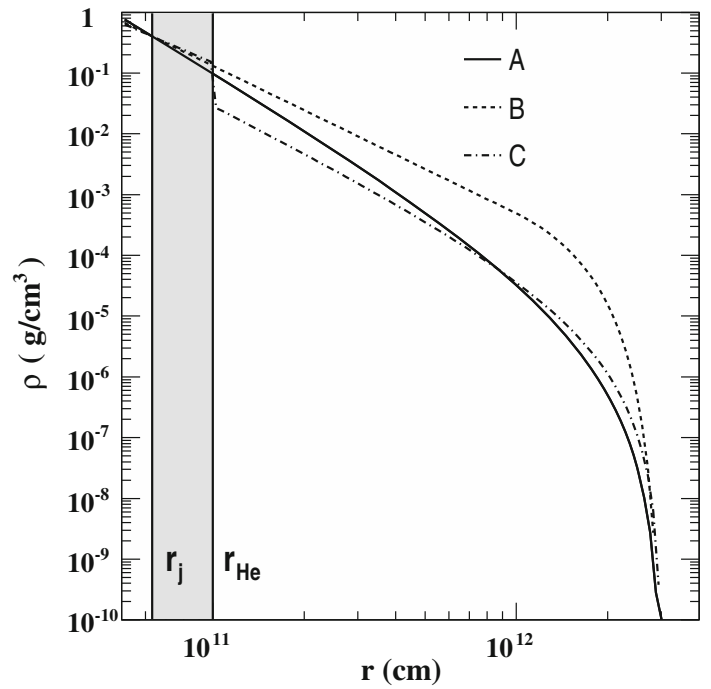

Fig. 6 Density profiles of the progenitor star taken from [17-20]. The density profiles $[A],[B],[C]$ are described in detail in [9]

before undergoing deep inelastic collisions with the surround medium to the detector, we must take into account the flavor oscillation.

In Paper I we have already used the analytic formalism developed by Ohlsson and Snellman (OS) to calculate threeflavor neutrino oscillations $[21,22]$ in the presupernova star [9] and then calculate the flavor ratio of neutrinos arriving on Earth. Here we extend the calculation by taking into account the matter effect of the Earth to calculate the flavor ratio at the IceCube detector. For this calculation we use the Earth density profile given in Eq. (4).

The input neutrino fluxes at the surface of the Earth, as functions of neutrino energy $E_{v}$, are those calculated and discussed thoroughly in Paper I [9], for three different models of the presupernova star, which we will refer to as model A, B, and C. For reference we present the density profile of these three models in Fig. 6. In Figs. 7 and 8 the neutrino and antineutrino fluxes at the detector, as functions of the neutrino energy, resulting from models A and B (in (b), (c), and (d)) and taking into account the Earth's matter effect, are compared with the case in which the effects of the stellar medium are ignored (in (a)). The two sets of plots, corresponding to different neutrino-mixing angles, $\theta_{13}$, are shown. In these plots the neutrinos have traversed the whole Earth before arriving at the detector (a $180^{\circ}$ zenith angle). All other parameters are taken from the best fit parameters from different experiments which are summarized in Table 1. We also consider two sets of parameters, Set I and Set II, corresponding to two different presuprenova star radii $R^{*}$ as shown in Table 1 and analyze our results.

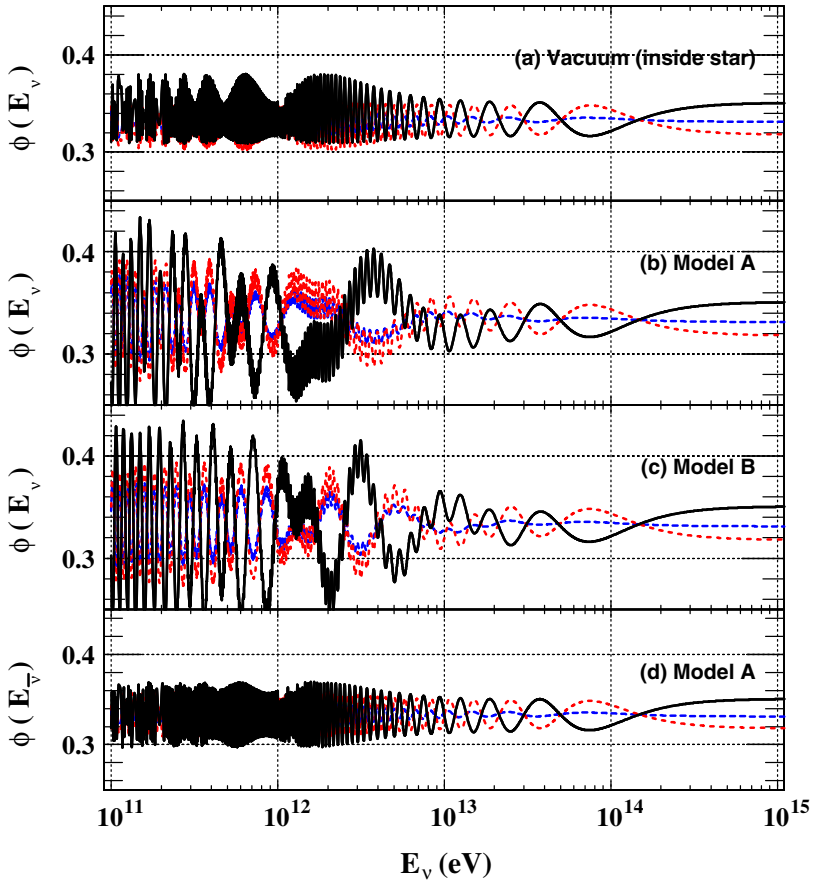

Fig. 7 Neutrino and antineutrino fluxes at the detector. In (a), (b), and (c) solid lines is for $\Phi_{v_{\mathrm{e}}}$, dashed lines are for $\Phi_{v_{\mu}}$, and dotted lines are for $\Phi_{\nu_{\tau}}$. In (d) solid line, dashed line and dotted line are for $\Phi_{\bar{\nu}_{\mathrm{e}}}$, $\Phi_{\bar{v}_{\mu}}$ and $\Phi_{\bar{v}_{\tau}}$ respectively. The neutrino-mixing parameters are: $\delta_{\mathrm{CP}}=$ $0 ; \theta_{12}=33.8^{\circ} ; \theta_{13}=8.8^{\circ} ; \theta_{23}=45.0^{\circ} ; \Delta m_{21}^{2}=8.0 \times 10^{-5} \mathrm{eV}^{2}$; $\Delta m_{23}^{2}=3.2 \times 10^{-3} \mathrm{eV}^{2}$

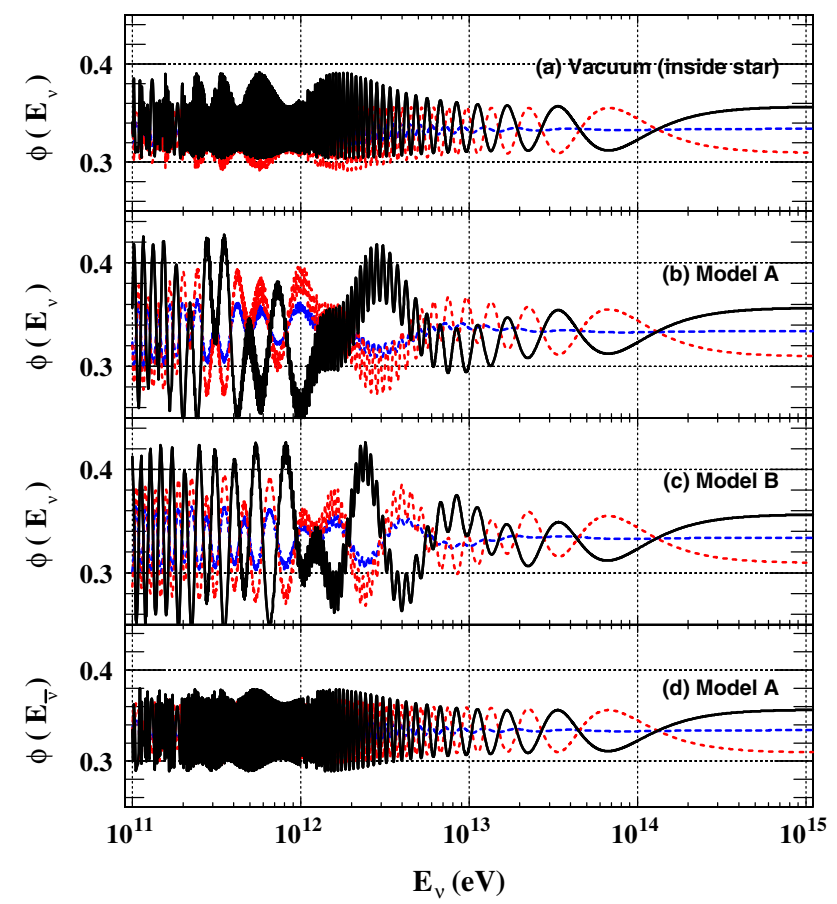

Fig. 8 Same as Fig. 7 but here we consider $\theta_{13}=12.0^{\circ}$ 
Table 1 The parameters considered for our study. $R^{*}$ is the radius of the presupernova star. We consider three different values of $\Delta m_{32}^{2}$ to observe the variation in $R$

\begin{tabular}{lll}
\hline Parameter & Set I & Set II \\
\hline$R^{*}$ & $3 \times 10^{12} \mathrm{~cm}$ & $2.7 \times 10^{12} \mathrm{~cm}$ \\
$\theta_{12}$ & $33.8^{\circ}$ & $33.8^{\circ}$ \\
$\theta_{13}$ & $8.8^{\circ}$ & $12^{\circ}$ \\
$\theta_{23}$ & $45^{\circ}$ & $45^{\circ}$ \\
$\Delta m_{21}^{2} / e V^{2}$ & $8.5 \times 10^{-5}$ & $8.5 \times 10^{-5}$ \\
$\Delta m_{32}^{2} / e V^{2}$ & $1.4 \times 10^{-3}$ & $1.4 \times 10^{-3}$ \\
& $3.2 \times 10^{-3}$ & $3.2 \times 10^{-3}$ \\
& $6.0 \times 10^{-3}$ & $6.0 \times 10^{-3}$ \\
\hline
\end{tabular}

\section{Detection of neutrinos by IceCube}

A neutrino detector, like IceCube, detects high energy neutrinos by observing the Cherenkov radiation emitted by the secondary charged particles produced when high energy neutrinos interact with the surrounding rock and ice [23]. These secondaries produce showers events and/or tracks events depending on the primary neutrino flavor. The neutrino interaction with rock and ice takes place through the neutral current (NC) and/or charge current (CC) weak processes $v_{l}+N \rightarrow v_{l}(l)+X$. In the NC case, since there is a neutrino in the final state, the only signature of the interaction will be through the hadronic shower, independent of the neutrino flavor. In the CC case the end-result depends on the neutrino flavor. If the interacting neutrino is an electron type, the resulting electron will quickly interact with the medium, producing an electromagnetic shower, which will overlap with the hadronic shower. If the neutrino is muon type, the resulting muon will produce a long track that emerges from the shower. Finally, if the neutrino is tau type, the resulting tau lepton may or may not produce a track depending on its energy. But when the tau decays into a muon, $\tau \rightarrow v_{\mu} \mu v_{\tau}$ the latter will produce a long track, just like in the case of a muon-neutrino $\mathrm{CC}$ interaction, this modifies the number of track events, which has to be accounted for. Since in this work we consider neutrinos coming from underneath the detector, those with energies above $1 \mathrm{PeV}$ will be drastically suppressed, and therefore the lollipop and double-bang events that are associated with very energetic $\nu_{\tau}$ will also be suppressed [24]. In this work we will not consider these kinds of events, however, we will include the $\mu$-track events induced by tau-neutrinos, as explained above.

In conclusion, the ratio of track events to shower events is related in a convoluted way to the neutrino flavor ratios. However, given a set of flavor ratios, like 1:1:1 in the "standard picture", or any other set, like in the case we are presenting in this work, the ratio of tracks-to-showers $R$ can be calculated.
In the next section we discuss in detail the track-to-shower ratio calculation.

\section{The track-to-shower ratio}

The calculation of the track-to-shower ratio $R$ presented in this section is based on the calculations from Refs. [24,25]. Here we have included the shadowing effect due to the neutrino absorption by the Earth, $P_{\text {shad }}\left(E_{v}, \theta\right)$. Since we are considering neutrinos coming from underneath, $\theta=180^{\circ}$, $P_{\text {shad }}\left(E_{v}\right)=P_{\text {shad }}\left(E_{v}, \theta=180^{\circ}\right)$. The ratio $R$ is defined as

$R=\frac{\text { Number of } \mu \text {-track events }}{\text { Number of shower-like events }}$.

The $\mu$-track events have two components: $N_{\mu \mu}$ from $\mu$-tracks induced by muon-neutrinos, and $N_{\mu \tau}$ from $\mu$-tracks induced by tau-neutrinos. The number of shower-like events have three components: $N_{\text {sh }_{\text {had }}}$ from hadronic showers associated with NC interaction, $N_{\mathrm{sh}_{\mathrm{em}}}$ from electromagnetic showers produced by CC interaction of $v_{\mathrm{e}}$, and $N_{\mathrm{sh}_{\tau}}$ from showers produced by CC interaction of $v_{\tau}$ decaying hadronically. So we can express $R$ as

$R=\frac{N_{\mu \mu}+N_{\mu \tau}}{N_{\mathrm{sh}_{\mathrm{had}}}+N_{\mathrm{sh}_{\mathrm{em}}}+N_{\mathrm{sh}_{\tau}}}$.

The $\mu$-tracks induced by $v_{\mu}\left(\bar{v}_{\mu}\right)$ result from the CC interaction of the neutrinos with the rock or the ice underground. The muons can travel a long distance before decaying; the effective muon range $R_{\mu}$ depends on the initial energy $E_{\mu}$ and the detection energy threshold $E_{\mu}^{\text {th }}$; in the case of IceCube this threshold is $\sim 100 \mathrm{GeV}$. The $\mu$-track induced by $\nu_{\tau}\left(\bar{v}_{\tau}\right)$ results from the decay of a $\tau$ produced in a CC interaction into a $\mu$; this decay has a probability density $f\left(E_{\tau}, E_{\mu}\right)$ and a branching ratio $B=17.8 \%$. The expressions for $N_{\mu \mu}$ and $N_{\mu \tau}$ are given by

$$
\begin{aligned}
N_{\mu \mu}= & \rho A N_{\mathrm{A}} \int_{E_{\mu}^{\mathrm{th}}}^{\infty} \int_{E_{\mu}^{\mathrm{th}}}^{E_{v_{\mu}}} R_{\mu}\left(E_{\mu}, E_{\mu}^{\mathrm{th}}\right) P_{\text {shad }}\left(E_{v_{\mu}}\right) \\
& \times \frac{\mathrm{d} F_{v_{\mu}}}{\mathrm{d} E_{v_{\mu}}} \frac{\mathrm{d} \sigma^{\mathrm{CC}}}{\mathrm{d} E_{\mu}} \mathrm{d} E_{\mu} \mathrm{d} E_{v_{\mu}}+\left[v_{\mu} \rightarrow \bar{v}_{\mu}\right], \\
N_{\mu \tau}= & B \rho A N_{\mathrm{A}} \int_{E_{\mu}^{\text {th }}}^{\infty} \int_{E_{\mu}^{\text {th }}}^{E_{v_{\tau}}} \int_{E_{\mu}^{\text {th }}}^{\frac{E_{\tau}}{2}(1+\beta)} R_{\mu}\left(E_{\mu}, E_{\mu}^{\mathrm{th}}\right) P_{\text {shad }}\left(E_{v_{\mu}}\right) \\
& \times \frac{\mathrm{d} F_{v_{\tau}}}{\mathrm{d} E_{v_{\tau}}} \frac{\mathrm{d} \sigma^{\mathrm{CC}}}{\mathrm{d} E_{\tau}} f\left(E_{\tau}, E_{\mu}\right) \mathrm{d} E_{\mu} \mathrm{d} E_{\tau} \mathrm{d} E_{v_{\tau}}+\left[v_{\tau} \rightarrow \bar{v}_{\tau}\right],
\end{aligned}
$$

where the muon range is defined as

$$
R_{\mu}\left(E_{\mu}, E_{\mu}^{\mathrm{th}}\right)=(2.6 \mathrm{Km}) \ln \left[\frac{2.0+4.2 \times 10^{-3} E_{\mu}}{2.0+4.2 \times 10^{-3} E_{\mu}^{\mathrm{th}}}\right],
$$


and its probability density is given by

$f\left(E_{\tau}, E_{\mu}\right) \simeq \frac{5}{3 E_{\tau}}-\frac{3 E_{\mu}^{2}}{E_{\tau}^{3}}+\frac{4 E_{\mu}^{3}}{3 E_{\tau}^{4}}$.

The expression for $f\left(E_{\tau}, E_{\mu}\right)$ is an approximation valid for $\beta \rightarrow 1(\gamma \gg 1)$, where $\beta=\sqrt{1-1 / \gamma^{2}}=\sqrt{1-\left(\frac{m_{\tau}}{E_{\tau}}\right)^{2}}$.

The number of shower-like events for the different kinds of processes are given by

$$
\begin{aligned}
& N_{\mathrm{sh}_{\mathrm{had}}}=\sum_{l=e, \mu, \tau} \rho A L N_{\mathrm{A}}\left[\int_{E_{\mathrm{sh}}^{\mathrm{th}}}^{\infty} P_{\text {shad }}\left(E_{v_{l}}\right) \frac{\mathrm{d} F_{\nu_{l}}}{\mathrm{~d} E_{v_{l}}} \sigma^{\mathrm{NC}} \mathrm{d} E_{v_{l}}\right. \\
& \left.+\int_{E_{\mathrm{sh}}^{\mathrm{th}}}^{\infty} P_{\text {shad }}\left(E_{\bar{\nu}_{l}}\right) \frac{\mathrm{d} F_{\bar{\nu}_{l}}}{\mathrm{~d} E_{\bar{\nu}_{l}}} \sigma^{\mathrm{NC}} \mathrm{d} E_{\bar{\nu}_{l}}\right] \\
& N_{\mathrm{sh}_{\mathrm{em}}}=\rho A L N_{\mathrm{A}}\left[\int_{E_{\mathrm{sh}}^{\mathrm{th}}}^{\infty} P_{\text {shad }}\left(E_{v_{\mathrm{e}}}\right) \frac{\mathrm{d} F_{\nu_{\mathrm{e}}}}{\mathrm{d} E_{v_{\mathrm{e}}}} \sigma^{\mathrm{CC}} \mathrm{d} E_{\nu_{\mathrm{e}}}\right. \\
& \left.+\int_{E_{\mathrm{sh}}^{\mathrm{th}}}^{\infty} P_{\text {shad }}\left(E_{\bar{\nu}_{\mathrm{e}}}\right) \frac{\mathrm{d} F_{\bar{\nu}_{\mathrm{e}}}}{\mathrm{d} E_{\bar{\nu}_{\mathrm{e}}}} \sigma^{\mathrm{CC}} \mathrm{d} E_{\bar{\nu}_{\mathrm{e}}}\right] \text {, } \\
& N_{\mathrm{sh}_{\tau}}=(1-B) \rho A L N_{\mathrm{A}}\left[\int_{E_{\mathrm{sh}}^{\mathrm{th}}}^{\infty} P_{\mathrm{shad}}\left(E_{v_{\tau}}\right) \frac{\mathrm{d} F_{v_{\tau}}}{\mathrm{d} E_{v_{\tau}}} \sigma^{\mathrm{CC}} \mathrm{d} E_{v_{\tau}}\right. \\
& \left.+\int_{E_{\mathrm{sh}}^{\mathrm{th}}}^{\infty} P_{\text {shad }}\left(E_{\bar{\nu}_{\tau}}\right) \frac{\mathrm{d} F_{\bar{\nu}_{\tau}}}{\mathrm{d} E_{\bar{\nu}_{\tau}}} \sigma^{\mathrm{CC}} \mathrm{d} E_{\bar{\nu}_{\tau}}\right] \text {, }
\end{aligned}
$$

where $\rho$ is the density of the detector medium, $A$ is the effective area of the detector, $L$ is the length of the detector, $N_{\mathrm{A}}$ is Avogadro's number and $\mathrm{d} F_{v_{l}} / \mathrm{d} E_{v_{l}}$ is defined in Eq. (1). The normalization for this equation, $N_{\nu_{l}}$, is proportional to the neutrino flux, for the different flavors. Since $\mathrm{d} F_{\nu_{l}} / \mathrm{d} E_{v_{l}}$ is evaluated in the quotient of Eq. (5), the proportionality constant cancels out. The total cross sections for $\mathrm{CC}\left(\sigma^{\mathrm{CC}}\right)$ and $\mathrm{NC}\left(\sigma^{\mathrm{NC}}\right)$ shown in Figs. 2 and 3 are used to evaluate $N_{\text {sh }}$ and $N_{\text {shem }_{\text {em }}}$.

In order to evaluate $\mathrm{d} \sigma^{\mathrm{CC}} / \mathrm{d} E_{l}$ we performed an empirical fit to the differential cross section presented in Fig. 4 of Ref. [26], which is given as

$\frac{1}{\sigma^{\mathrm{CC}}} \frac{\mathrm{d} \sigma^{\mathrm{CC}}}{\mathrm{d} y}=N_{0} \begin{cases}b_{1} y^{-a_{1}} & \text { if } y<y_{\text {cut }} \\ b_{2} y^{-a_{2}} & \text { if } y \geq y_{\text {cut }},\end{cases}$

where $N_{0}$ is the normalization,

$y=\frac{E_{v_{l}}-E_{l}}{E_{v_{l}}}$

and

$y_{\mathrm{cut}}=\exp \left(\frac{\log b_{1}-\log b_{2}}{a_{1}-a_{2}}\right)$.

The parameters in Eq. (14) are as follows:

$a_{1}=-0.0163 x^{2}+0.3877 x-1.1905$,

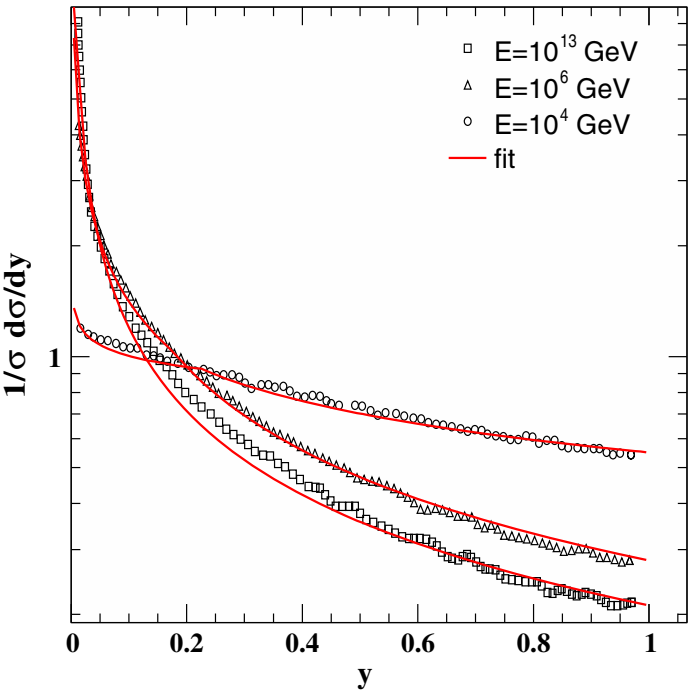

Fig. 9 Comparison between our empirical fit, based on Eq. (14), and the Monte Carlo results presented in Ref. [26]

$a_{2}=-0.0222 x^{2}+0.4222 x-0.9833$,

$b_{1}=0.0168 x^{2}-0.3683 x+2.0038$

$b_{2}=0.0139 x^{2}-0.2739 x+1.4233$,

and

$x=\log _{10}\left(E_{v_{l}} / \mathrm{GeV}\right)$.

The normalization is set such that

$\int_{0}^{1}\left(\frac{1}{\sigma^{\mathrm{CC}}} \frac{\mathrm{d} \sigma^{\mathrm{CC}}}{\mathrm{d} y}\right) \mathrm{d} y=1$.

We compare our fit with the data presented in Ref. [26] which are shown in Fig. 9.

After performing the necessary change of variable from $E_{l}$ to $y$, one can evaluate the integrals numerically. The neutrinoflavor ratios, $R$, obtained after propagating the neutrinos from the source, all the way up to the detector, for different combinations of the parameters involved, and for different energies, are used as input for the calculation.

\section{Results}

As can be seen from Figs. 7 and 8, the normalized flux of neutrinos and antineutrinos in the detector depends on energy. For the calculation of the ratio $R$ we need the neutrino flux $\mathrm{d} F_{v} / \mathrm{d} E_{v}$. We neither know the exact form of it nor the spectral index $\alpha$. But by considering the neutrino flux ratio 1:2:0 at the source, then propagating these neutrinos through the presupernova matter we calculated the normalized flux on the surface of the star in Paper I. Here, we take this normalized flux and propagate the neutrinos through the dis- 


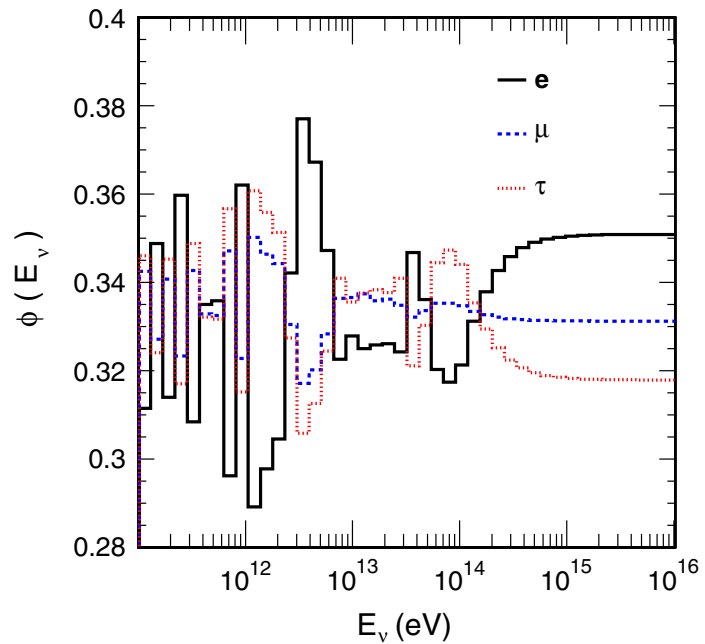

Fig. 10 Neutrino flux $\Phi_{v}\left(E_{v}\right)$ as a function of neutrino energy $E_{v}$ in the detector

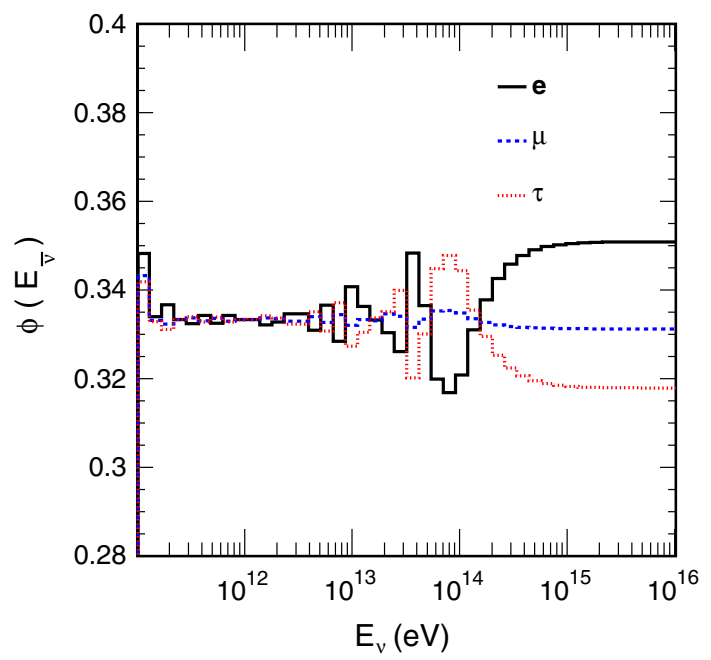

Fig. 11 Antineutrino flux $\Phi\left(E_{\bar{v}}\right)$ as a function of the neutrino energy $E_{v}$ in the detector

tance between the source and the Earth, where the Earth's matter effect is included and we calculate the normalized flux of these neutrinos and antineutrinos in the detector. For the calculation of the track-to-shower ratio $R$ of Eq. (5) we use these fluxes. But instead of calculating the flux for each energy, we divide the whole energy range to energy bins as $\Delta E_{v}=0.3 E_{v}$ i.e. $30 \%$ energy resolution. Within each bin the flux is constant, which we take by averaging the flux in the same energy bin. Here we have shown these average neutrino and antineutrino fluxes in Figs. 10 and 11. From these figures, it is observed that the average neutrino and antineutrino fluxes are different for $E_{v}<2 \times 10^{13} \mathrm{eV}$. Finally, we consider two values of the $\mathrm{CP}$ violating phase $\delta_{\mathrm{CP}}=0$ and $\pi$ to see the change in $R$. The upper limit of the $E_{v}$ is taken to be $10 \mathrm{PeV}$ to evaluate the neutrino energy integrals. The following values are considered for the IceCube detector in

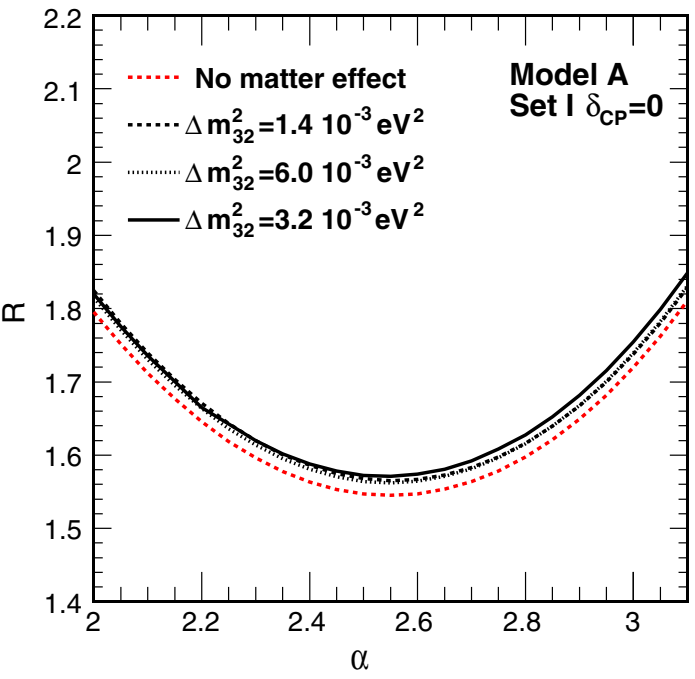

Fig. 12 The track-to-shower ratio $R$ as a function of the spectral index $\alpha$ for $\delta_{\mathrm{CP}}=0$ in model $\mathrm{A}$

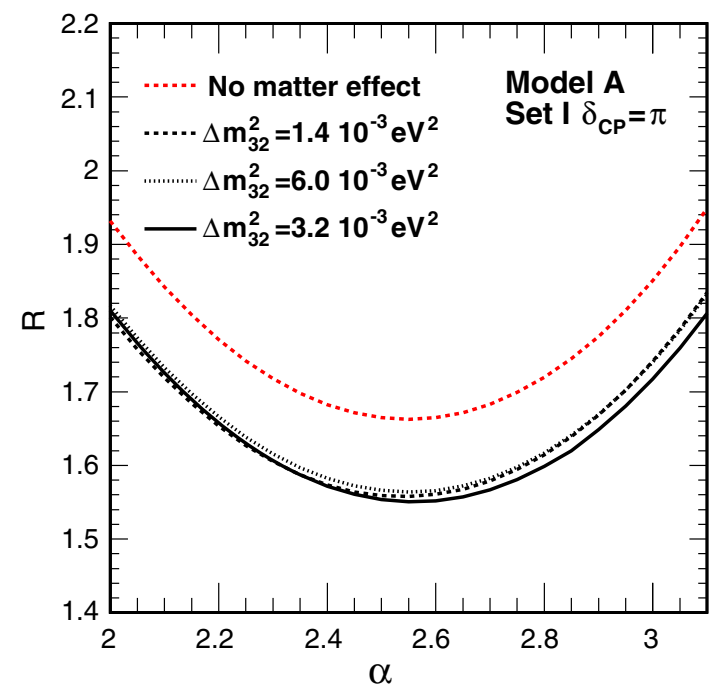

Fig. 13 The track-to-shower ratio $R$ as a function of the spectral index $\alpha$ for $\delta_{\mathrm{CP}}=\pi$ in model $\mathrm{A}$

our calculation: density of ice $\rho=0.051 \mathrm{~g} \mathrm{~cm}^{-3}$, detector area $A=10^{10} \mathrm{~cm}^{2}$, and the detector length $L=10^{5} \mathrm{~cm}$. The results are presented in Figs. 12, 13, 14, 15, 16, 17, 18, 19, 20.

In Figs. 12, 13, 14, 15, we show $R$ as a function of the spectral index $\alpha$ for models A and C. In these figures we also include no matter effect which implies the following. At the source we consider the flux ratio 1:2:0 and these neutrinos propagate up to the detector in vacuum. For convenience we define the track-to-shower ratio for the case of no matter effect as $R_{0}$. For $\delta_{\mathrm{CP}}=0$ we found that $R_{0} \leq R$ for any given value of $\alpha$. Also the gap between $R$ and $R_{0}$ is small. On the other hand, for $\delta_{\mathrm{CP}}=\pi$, we always found $R_{0}>R$ and the gap is bigger. The value of $R$ is minimum around 


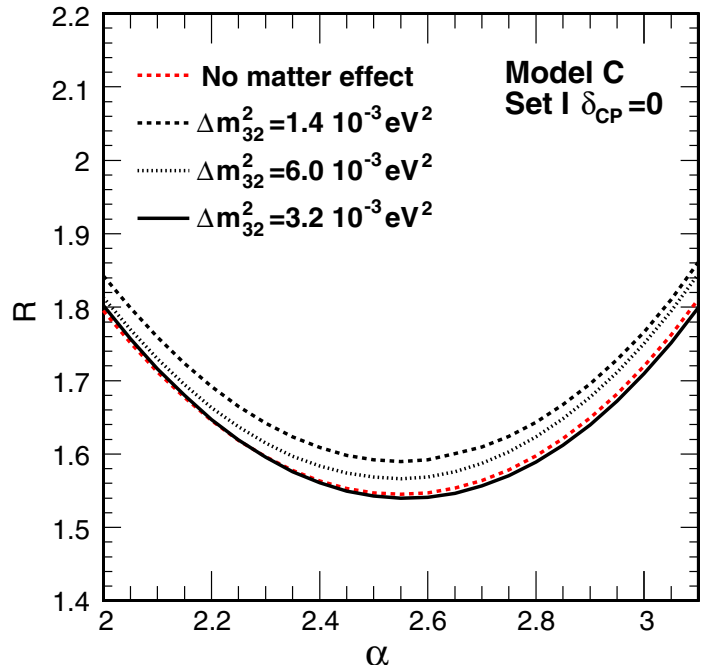

Fig. 14 Same as Fig. 12 for model C

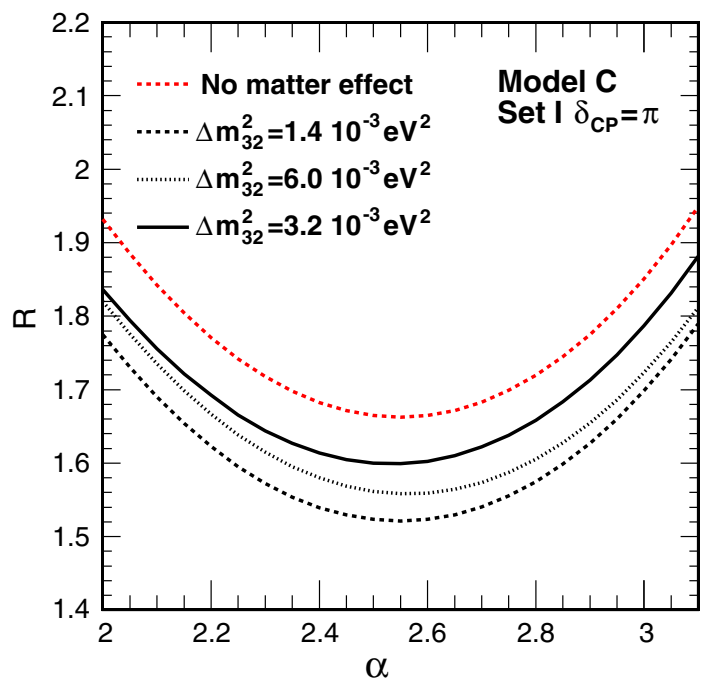

Fig. 15 Same as Fig. 13 for model C

$\alpha=2.6$, which is independent of whether we consider the matter effect or not. We also show results for three different $\Delta m_{32}^{2}$ values, which shows that there is very little variation in $R$. This minimum value of $R$ is also independent of $\Delta m_{32}^{2}$. The order in which $R$ is arranged for different $\Delta m_{32}^{2}$ values reverses by going from $\delta_{\mathrm{CP}}=0$ to $\pi$, which can be seen by comparing Fig. 12 with Fig. 13 in model A and similarly Fig. 14 with Fig. 15 in model C. Here we have omitted the results from model $\mathrm{B}$ because the results are very similar to model A.

In Figs. 16, 17, 18, 19 we show the variation of $R$ as a function of $\sin ^{2} \theta_{13}$ in models $\mathrm{A}$ and $\mathrm{C}$ for three different values of the spectral index $\alpha$. In these plots we observe that the ratio $R$ is almost constant for a given $\alpha$ and for both $\delta_{\mathrm{CP}}=0$ and $\pi$, as we vary $\sin ^{2} \theta_{13}$ for all the models. Also the value of $R$ is higher for smaller $\alpha$.

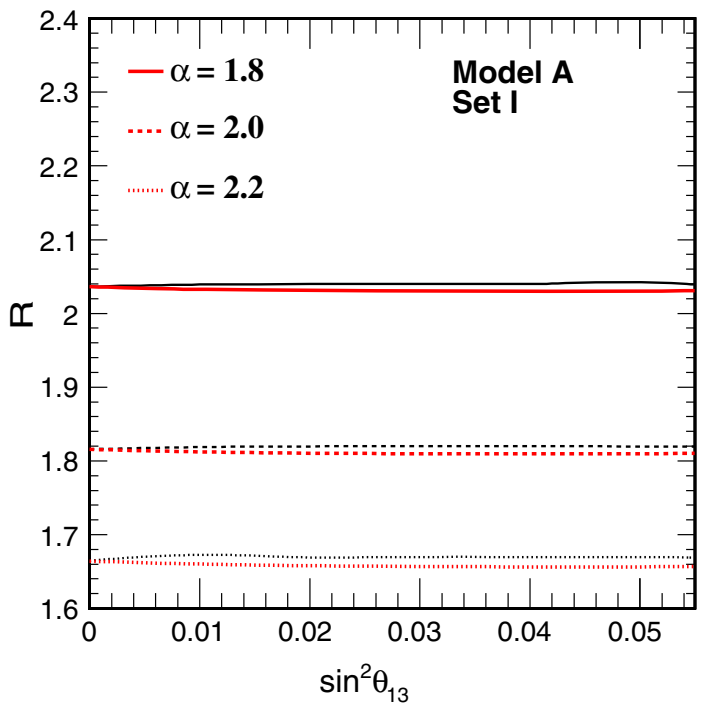

Fig. 16 The track-to-shower ratio $R$ as a function of $\sin ^{2} \theta_{13}$ in model A for the parameter Set I with $\Delta m_{32}^{2}=3.2 \times 10^{-3} \mathrm{eV}^{2}$. The black curve is for $\delta_{\mathrm{CP}}=0$ and the red one is for $\delta_{\mathrm{CP}}=\pi$

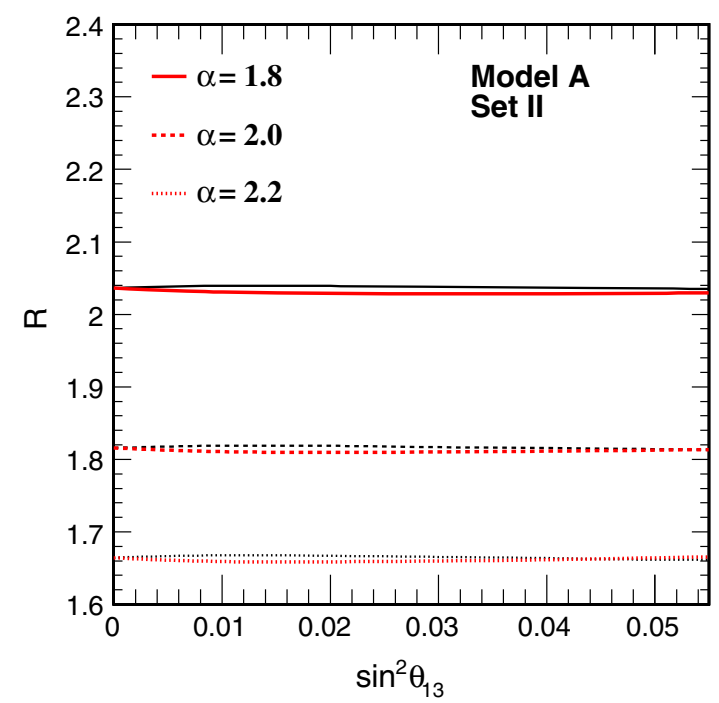

Fig. 17 The track-to-shower ratio $R$ as a function of $\sin ^{2} \theta_{13}$ in model A for the parameter Set II with $\Delta m_{32}^{2}=3.2 \times 10^{-3} \mathrm{eV}^{2}$. The black curve is for $\delta_{\mathrm{CP}}=0$ and the red one is for $\delta_{\mathrm{CP}}=\pi$

We also show $R$ as a function of $\sin ^{2} \theta_{13}$ for the case of no matter effect in Fig. 20. This shows a clear difference between $\delta_{\mathrm{CP}}=0$ (lower curve) and $\delta_{\mathrm{CP}}=\pi$ (upper curve) for each $\alpha$. These two curves diverge from the point $\theta_{13}=0$ as can be seen from the plots in Fig. 20. Comparison of the matter effect (from Figs. 16, 17, 18, 19) with the no matter effect Fig. 20 shows that the $\delta_{\mathrm{CP}}=\pi$ contribution is very much suppressed in matter compared to the $\delta_{\mathrm{CP}}=0$ contribution and it makes them almost the same. In the mixing matrix, the terms containing $\mathrm{CP}$ phases will change sign going from $\delta_{\mathrm{CP}}=0$ to $\delta_{\mathrm{CP}}=\pi$ and in the vacuum case this will split the 


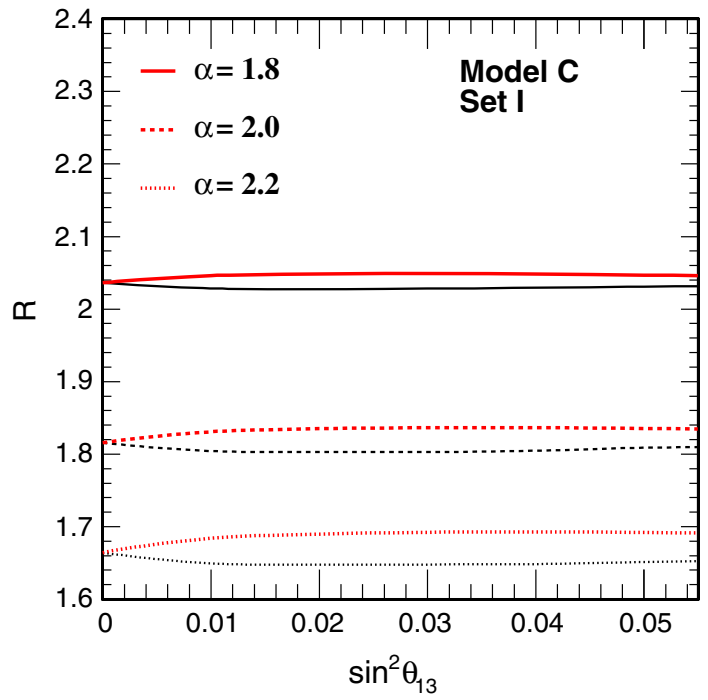

Fig. 18 The track-to-shower ratio $R$ as a function of $\sin ^{2} \theta_{13}$ in model $\mathrm{C}$ for the parameter Set I with $\Delta m_{32}^{2}=3.2 \times 10^{-3} \mathrm{eV}^{2}$. The black curve is for $\delta_{\mathrm{CP}}=0$ and the red one is for $\delta_{\mathrm{CP}}=\pi$

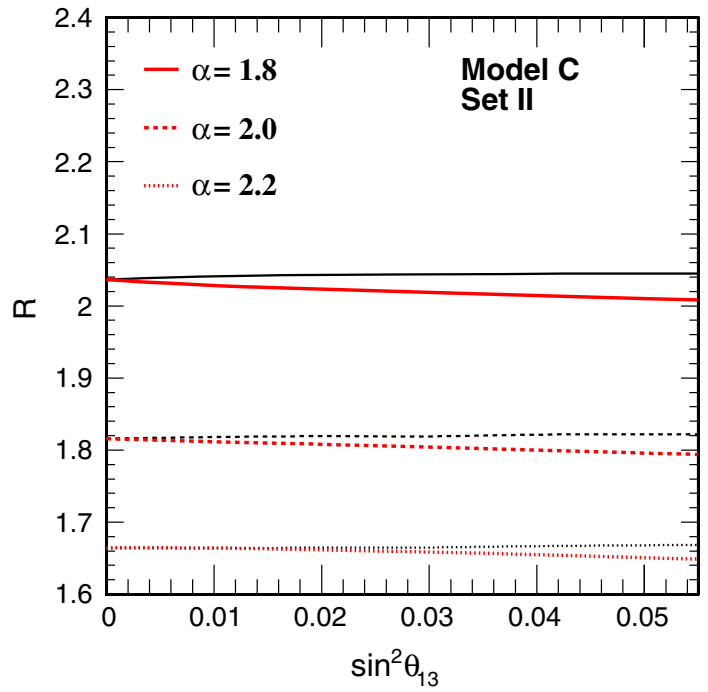

Fig. 19 The track-to-shower ratio $R$ as a function of $\sin ^{2} \theta_{13}$ in model $\mathrm{C}$ for the parameter Set II with $\Delta m_{32}^{2}=3.2 \times 10^{-3} \mathrm{eV}^{2}$. The black curve is for $\delta_{\mathrm{CP}}=0$ and the red one is for $\delta_{\mathrm{CP}}=\pi$

ratio $R$ by lifting up the $\delta_{\mathrm{CP}}=\pi$ contribution as shown in Fig. 20. This enhancement is being compensated by the matter effect, so that we do not have two pronouncedly different curves for $\delta_{\mathrm{CP}}=0$ and $\delta_{\mathrm{CP}}=\pi$ with matter contribution as shown in Figs. 16, 17, 18, 19. This shows that the trackto-shower ratio $R$ for high energy neutrinos in IceCube is probably almost blind to the $\mathrm{CP}$ violating phases when the Earth matter effect is taken into account.

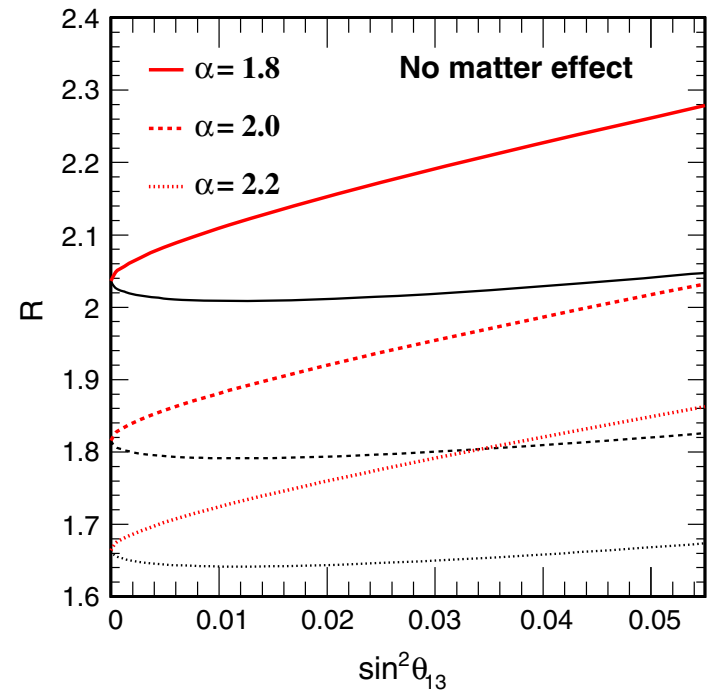

Fig. 20 The track-to-shower ratio $R=R_{0}$ (as described in the text) as a function of $\sin ^{2} \theta_{13}$ with no matter effect. Here also the black curve is for $\delta_{\mathrm{CP}}=0$ and the red one is for $\delta_{\mathrm{CP}}=\pi$. We take $\Delta m_{32}^{2}=$ $3.2 \times 10^{-3} \mathrm{eV}^{2}$

\section{Summary}

A very small fraction $\left(\leq 10^{-3}\right)$ of the core collapse supernovae can produce GRBs by launching a successful jet. Although the majority of these core collapses cannot produce GRBs, very high energy neutrinos can easily be produced in their choked jets. These neutrinos propagating through the over-burden matter can undergo oscillation and the flux ratio on the surface of the star can be different from the point where these neutrinos were produced. Along the Mpc long baseline, from the surface of the star to the surface of the Earth, these neutrinos will have vacuum oscillation. Before reaching the detector from the opposite side of the Earth, these neutrinos will cross the diameter of the Earth and again can undergo matter oscillation. By considering a realistic density profile of the Earth we have extended our previous work to study numerically the three neutrino oscillation and evaluate the change in the flux ratio in the detector. Depending on the energy of these neutrinos, there can also be a shadowing effect and neutrinos above a few $\mathrm{PeV}$ can be completely absorbed. In this work we have done a thorough analysis of the high energy neutrino propagation in the Earth before reaching the detector by taking into account the shadowing effect. The track-to-shower ratio $R$ is calculated for these high energy neutrinos. In the calculation of $R$ we have included the shadowing effect and the contribution of the muon track produced by the high energy $\tau$ lepton decay around the IceCube detector. These $\tau$ leptons are produced due to the CC interaction of $v_{\tau}$ with the surround rock and ice of the detector. We have studied the variation of $R$ when the spectral index $\alpha$ and the mixing angle $\sin ^{2} \theta_{13}$ vary. We 
found that $R$ has a minimum around $\alpha=2.6$ and is independent of whether we consider the matter effect or not. This minimum value of $R$ is also independent of the $\Delta m_{32}^{2}$ value. We observed that the ratio $R$ is different for $\delta_{\mathrm{CP}}=0$ and $\pi$ when no matter effect is considered. But when the Earth matter contribution is taken into account, the $R$ value is almost blind to these different $\mathrm{CP}$ phases.

Acknowledgments S.S. is thankful to Departamento de Fisica de Universidad de los Andes, Bogota, Colombia, for kind hospitality during several visits. This work is partially supported by DGAPA-UNAM (Mexico) Project No. IN103812.

Open Access This article is distributed under the terms of the Creative Commons Attribution 4.0 International License (http://creativecomm ons.org/licenses/by/4.0/), which permits unrestricted use, distribution, and reproduction in any medium, provided you give appropriate credit to the original author(s) and the source, provide a link to the Creative Commons license, and indicate if changes were made. Funded by SCOAP ${ }^{3}$.

\section{References}

1. B. Zhang, P. Meszaros, Int. J. Mod. Phys. A 19, 2385 (2004). arXiv:astro-ph/0311321

2. C. Kouveliotou, C.A. Meegan, G.J. Fishman, N.P. Bhyat, M.S. Briggs, T.M. Koshut, W.S. Paciesas, G.N. Pendleton, Astrophys. J. 413, L101 (1993)

3. E. Waxman, J.N. Bahcall, Phys. Rev. Lett. 78, 2292 (1997)

4. E. Waxman, J.N. Bahcall, Phys. Rev. D 59, 023002 (1999)

5. E. Berger, S.R. Kulkarni, D.A. Frail, A.M. Soderberg, Astrophys. J. 599, 408 (2003)

6. S.E. Woosley, J.S. Bloom, Ann. Rev. Astron. Astrophys. 44, 507 (2006). arXiv:astro-ph/0609142

7. P.A. Mazzali, J.S. Deng, N. Tominaga, K. Maeda, K. Nomoto, T. Matheson, K.S. Kawabata, K.Z. Stanek et al., Astrophys. J. 599, L95 (2003). arXiv:astro-ph/0309555
8. S. Sahu, B. Zhang, Res. Astron. Astrophys. 10, 943 (2010). arXiv:1007.4582 [hep-ph]

9. A.F. Osorio Oliveros, S. Sahu, J.C. Sanabria, Eur. Phys. J. C 73, 2574 (2013). arXiv:1304.4906 [astro-ph]

10. M.G. Aartsen et al., IceCube Collaboration, Phys. Rev. Lett. 111, 021103 (2013). arXiv:1304.5356 [astro-ph.HE]

11. M.G. Aartsen et al., IceCube Collaboration, Science 342(6161), 1242856 (2013). arXiv:1311.5238 [astro-ph.HE]

12. M.G. Aartsen et al., IceCube Collaboration. arXiv:1405.5303 [astro-ph.HE]

13. M.G. Aartsen et al., IceCube Collaboration. arXiv:1410.1749 [astro-ph.HE]

14. F. Halzen, D.W. Hooper, Astrophys. J. 527, L93 (1999). arXiv:astro-ph/9908138

15. J.K. Becker, Phys. Rep. 458, 173 (2008). arXiv:0710.1557 [astro$\mathrm{ph}]$

16. R. Gandhi, C. Quigg, M.H. Reno, I. Sarcevic, Phys. Rev. D 58, 093009 (1998). arXiv:hep-ph/9807264

17. S.E. Woosley, A. Heger, T.A. Weaver, Rev. Mod. Phys. 74, 1015 (2002)

18. S.E. Woosley, N. Langer, T.A. Weaver, Astrophys. J. 448, 315 (1995)

19. T. Shigeyama, K. Nomoto, Astrophys. J. 360, 242 (1990)

20. O. Mena, I. Mocioiu, S. Razzaque, Phys. Rev. D 75, 063003 (2007). arXiv:astro-ph/0612325

21. T. Ohlsson, H. Snellman, J. Math. Phys. 41, 2768 (2000). (Erratumibid. 42, 2345 (2001)). arXiv:hep-ph/9910546

22. T. Ohlsson, H. Snellman, Eur. Phys. J. C 20, 507 (2001). arXiv:hep-ph/0103252

23. See IceCube website: http://icecube.wisc.edu/

24. J.F. Beacom, N.F. Bell, D. Hooper, S. Pakvasa, T.J. Weiler, Phys. Rev. D 68, 093005 (2003). (Erratum-ibid. D 72, 019901 (2005)). arXiv:hep-ph/0307025

25. A. Esmaili, Y. Farzan, Nucl. Phys. B 821, 197 (2009). arXiv:0905.0259 [hep-ph]

26. S. Bevan, S. Danaher, J. Perkin, S. Ralph, C. Rhodes, L. Thompson, T. Sloan, D. Waters, Astropart. Phys. 28, 366 (2007). arXiv:0704.1025 [astro-ph] 